\title{
Glycyrrhetinic acid induces G1-phase cell cycle arrest in human non-small cell lung cancer cells through endoplasmic reticulum stress pathway
}

\author{
JIE ZHU $^{1 *}$, MEIJUAN CHEN ${ }^{2 *}$, NING CHEN ${ }^{1}$, AIZHEN MA $^{1}$, CHUNYAN ZHU $^{1}$, RUOLIN ZHAO $^{2}$, \\ MIAO JIANG ${ }^{2}$, JING ZHOU ${ }^{2}$, LIHONG YE ${ }^{1}$, HAIAN FU $^{3,4}$ and XU ZHANG ${ }^{2}$ \\ ${ }^{1}$ The First Clinical Medicine College and ${ }^{2}$ Jiangsu Collaborative Innovation Center of \\ Traditional Chinese Medicine (TCM) Prevention and Treatment of Tumor, Nanjing University of Chinese Medicine, \\ Nanjing 210023, P.R. China; Departments of ${ }^{3}$ Pharmacology and ${ }^{4}$ Hematology \& Medical Oncology, \\ Emory University School of Medicine, Atlanta, GA 30322, USA
}

Received August 19, 2014; Accepted November 20, 2014

DOI: 10.3892/ijo.2015.2819

\begin{abstract}
Glycyrrhetinic acid (GA) is a natural compound extracted from liquorice, which is often used in traditional Chinese medicine. The purpose of the present study was to investigate the antitumor effect of GA in human non-small cell lung cancer (NSCLC), and its underlying mechanisms in vitro. We have shown that GA suppressed the proliferation of A549 and NCI-H460 cells. Flow cytometric analysis showed that GA arrested cell cycle in G0/G1 phase without inducing apoptosis. Western blot analysis indicated that GA mediated G1-phase cell cycle arrest by upregulation of cyclin-dependent kinase inhibitors (CKIs) (p18, p16, p27 and p21) and inhibition of cyclins (cyclin-D1, -D3 and -E) and cyclin-dependent kinases (CDKs) (CDK4, 6 and 2). GA also maintained pRb phosphorylation status, and inhibited E2F transcription factor 1 (E2F-1) in both cell lines. GA upregulated the unfolded proteins, Bip, PERK and ERP72. Accumulation of unfolded proteins in the endoplasmic reticulum (ER) triggered the unfolded protein response (UPR), which could be the mechanism by which GA inhibited cell proliferation in NSCLC cells. GA then coordinated the induction of ER chaperones, which decreased protein synthesis and induced cell cycle arrest in the G1 phase. This study provides experimental evidence to support the development of GA as a chemotherapeutic agent for NSCLC.
\end{abstract}

Correspondence to: Dr Xu Zhang, Jiangsu Collaborative Innovation Center of Traditional Chinese Medicine (TCM) Prevention and Treatment of Tumor, Nanjing University of Chinese Medicine, Nanjing 210023, P.R. China

E-mail: zhangxutcm@yahoo.com

*Contributed equally

Key words: glycyrrhetinic acid, cell cycle arrest, ER stress, NSCLC

\section{Introduction}

Lung cancer (LC) is an important etiology of malignant mortality worldwide with global statistics indicating $>1,000,000$ deaths each year (1). Non-small cell lung cancer (NSCLC) accounts for $80-85 \%$ of LC (2). The 5-year survival rate is $\sim 6.6 \%$ for advanced stage LC (stage III or IV) in the US (3). Apart from surgery, adjuvant chemotherapy with gefitinib, erlotinib, and epidermal growth factor receptor tyrosine kinase inhibitors (EGFR-TKIs), has been widely used to clinically treat NSCLC. However, their efficacy is limited because of natural or acquired resistance (4). Therefore, there is a need to identify and develop potential anticancer drugs with increased selectivity and reduced toxicity.

Glycyrrhetinic acid (GA) is a bioactive component of glycyrrhiza (GL), which is often used in Chinese traditional medicine to treat various diseases. GA is known to possess anti-inflammatory, anti-viral and cytokine-inducing activity (5-10). Recently, the antitumor activity of GA has been extensively studied. GA has been reported to have cytotoxic effects against human ovarian cancer, hepatocellular carcinoma, breast cancer, pituitary adenoma and human bladder cancer (11-16). However, no inhibitory activity on the growth of NCSLC cell lines has been reported.

Endoplasmic reticulum (ER) stress responses are mediated by the activation of several unfolded protein response (UPR)-signaling pathways. In mammalian cells, the UPR signals increase expression of ER chaperone proteins GRP78/Bip, GRP94, and CHOP (17). The UPR coordinates the induction of ER chaperones, which decreases protein synthesis and results in growth arrest in G1 phase of the cell cycle. Previous studies have demonstrated that ER stress triggers G1-phase cell cycle arrest in various cancer cells (18). However, the molecular mechanism underlying UPR-induced G1 cell cycle arrest remains largely unknown.

In this study, we investigated the effect of GA on survival and proliferation of human NSCLC cell lines (A549 and NCI-H460), and found that GA could suppress the proliferation of both cell lines, with A549 being more sensitive than 
NCI-H460. GA arrested cells in G1 phase via inactivation of CDK4/6-cyclin-D1/D3 complex through p18/p16 activation, and inactivation of CDK2-cyclin-E2 complex through p27/p21 activation. This resulted in $\mathrm{pRb}$ dephosphorylation and inactivation of E2F transcription factor 1 (E2F-1) in both cell types. E2F-1 is an essential transcription factor that regulates cell cycle progression and apoptosis. Additionally, GA was found to increase the expression of Bip, protein kinase-like ER kinase (PERK) and ERP72, which are linked to ER stress.

\section{Materials and methods}

Reagents. GA was purchased from Nanjing Zelang Medical Technology Co., Ltd. (Jiangsu, China), and dissolved in dimethyl sulfoxide (DMSO) (Sigma, St. Louis, MO, USA) to make a stock solution before use. For treatment of cells, it was diluted in culture medium at the appropriate concentrations, and the final concentration of DMSO was $<0.01 \%$ (v/v). Cisplatin (Lot no. H20030675; Nanjing Pharmaceutical Factory Co., Ltd., Jiangsu, China), and insulin, propidium iodide (PI), 3-(4,5-dimethylthiazol-2-yl)-2,5-diphenyltetrazolium bromide (MTT), and alamarBlue were from Sigma. Alexa Fluor 488 Annexin V/Dead Cell Apoptosis kit was from Invitrogen Life Technologies (Carlsbad, CA, USA). Antibodies against caspase-3, -7 and -9, p18, p16, p27, p21, cyclin-D1, -D3 and -E2, CDK6, 4 and 2, E2F-1, pRb, Bip, PERK, ERP72, $\beta$-actin, and HRP-conjugated antibodies (anti-rabbit or mouse immunoglobulin G) were obtained from Cell Signaling Technology, Inc. (Danvers, MA, USA).

BCA protein estimation kit was from Sigma. Nitrocellulose (NC) blotting membrane was from Pall Corporation (DF Mexico, Mexico). Enhanced chemiluminescence (ECL) was from Bio-Rad (Hercules, CA, USA).

Cell culture. Human NSCLC cell lines A549 and NCI-H460 were purchased from the Type Culture Collection of the Chinese Academy of Sciences (Shanghai, China). A549 cells were cultured in DMEM/F12 (Gibco-BRL, Carlsbad, CA, USA), supplemented with $10 \%$ fetal bovine serum (FBS). NCI-H460 cells were grown in RPMI-1640 medium (Gibco-BRL), supplemented with 10\% FBS. All cells were cultured under $5 \% \mathrm{CO}_{2}$ at $37^{\circ} \mathrm{C}$.

In vitro viability assay. The effect of GA on cell viability was measured using the MTT assay. Cells were seeded in 96-well plates at $5 \times 10^{3}$ cells/well in $100 \mu \mathrm{l}$ of culture medium, and treated with drug the next day for 24,48 , and $72 \mathrm{~h}$. The final concentrations of GA used in the assays were 50, 25, 12.5, 6.25 and $3.125 \mu \mathrm{mol} / 1$ in triplicate, respectively. Treated cells were incubated with $20 \mu \mathrm{l}$ of MTT $(5 \mathrm{mg} / \mathrm{ml})$ for $4 \mathrm{~h}$ at $37^{\circ} \mathrm{C}$ in the dark. Optical density of producer after incubation was measured using a microplate reader (Bio-Rad) at a wavelength of $490 \mathrm{~nm}$.

Cell cycle analysis. After treatment with various concentrations of GA for different time, the cells were harvested with trypsin, washed once with PBS, and then fixed in 70\% ethanol overnight at $4^{\circ} \mathrm{C}$. Before flow cytometry analysis, the cells were then treated with $1 \mathrm{mg} / \mathrm{ml}$ of RNase for $30 \mathrm{~min}$ at $37^{\circ} \mathrm{C}$, and then stained with $40 \mu \mathrm{g} / \mathrm{ml}$ of PI for $30 \mathrm{~min}$. A total of $1 \times 10^{4}$ cells/sample were analyzed using a FACSCalibur flow
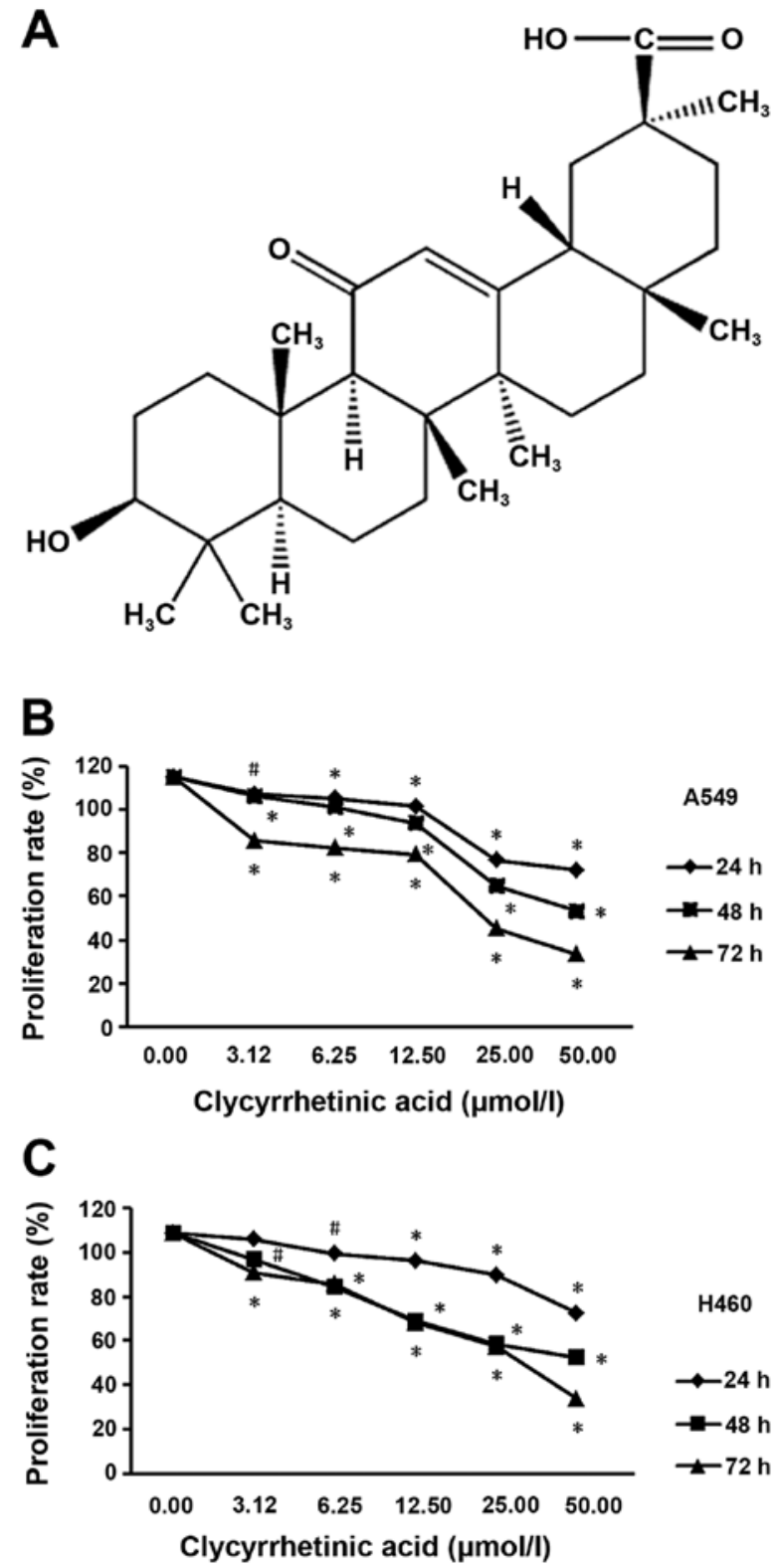

Figure 1. Dose- and time-dependent effect of glycyrrhetinic acid (GA) on cell viability in non-small cell lung cancer (NSCLC) cell lines. (A) Structural formula of GA. (B) Viability of A549 and (C) of NCI-H460 cells at 24, 48 and $72 \mathrm{~h}$ after treatment with increasing doses of GA. Actual cell numbers were counted with alamarBlue dye. Results shown are the mean of three independent experiments. Error bars represent SD; ${ }^{*} \mathrm{p}<0.01,{ }^{\#} \mathrm{p}<0.05$. GA induces cell cycle arrest at G1 phase in NSCLC cells without induction of apoptosis.

cytometer (BD Biosciences, Heidelberg, Germany). Data were evaluated using ModFit software.

Western blot analysis. After treatment with different concentrations of GA, the cells were lysed in RIPA buffer containing $50 \mathrm{mM}$ Tris/ $\mathrm{HCl}(\mathrm{pH} 8.0), 150 \mathrm{mM} \mathrm{NaCl}, 1 \%(\mathrm{w} / \mathrm{v})$ Nonidet P-40, $1 \%(\mathrm{w} / \mathrm{v})$ sodium deoxycholate, $0.1 \%(\mathrm{w} / \mathrm{v})$ SDS, $0.1 \mathrm{mM}$ DTT, $0.05 \mathrm{mM}$ PMSF, $0.002 \mathrm{mg} / \mathrm{ml}$ aprotinin, $0.002 \mathrm{mg} / \mathrm{ml}$ leupeptin, and $1 \mathrm{mM} \mathrm{NaVO}$. The protein concentrations of the supernatants were determined by the BCA Protein Assay kit. Equal amounts of the protein were loaded and separated by 10 or $12 \%$ SDS-PAGE, and then transferred onto NC membranes. The membranes were incubated overnight 
A

$24 \mathrm{~h}$

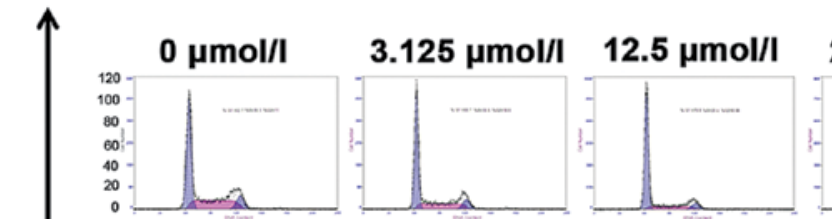

$48 \mathrm{~h}$

$72 \mathrm{~h}$
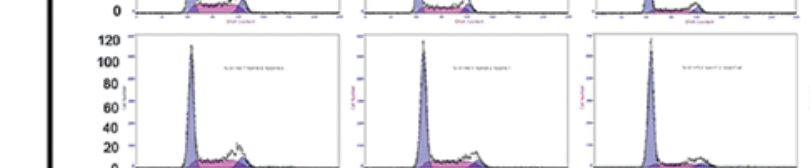

$25 \mu \mathrm{mol} / \mathrm{I}$

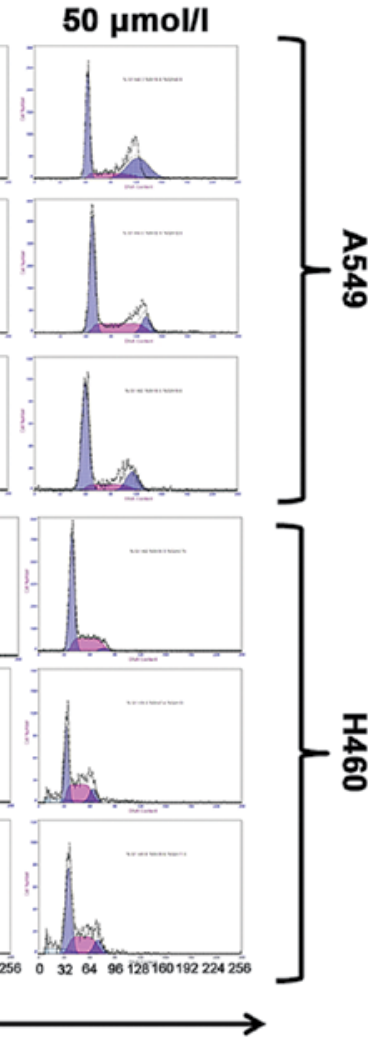

$24 \mathrm{~h}$

$48 \mathrm{~h}$

$72 \mathrm{~h}$

$\prod_{0}$

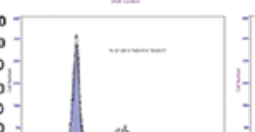

La
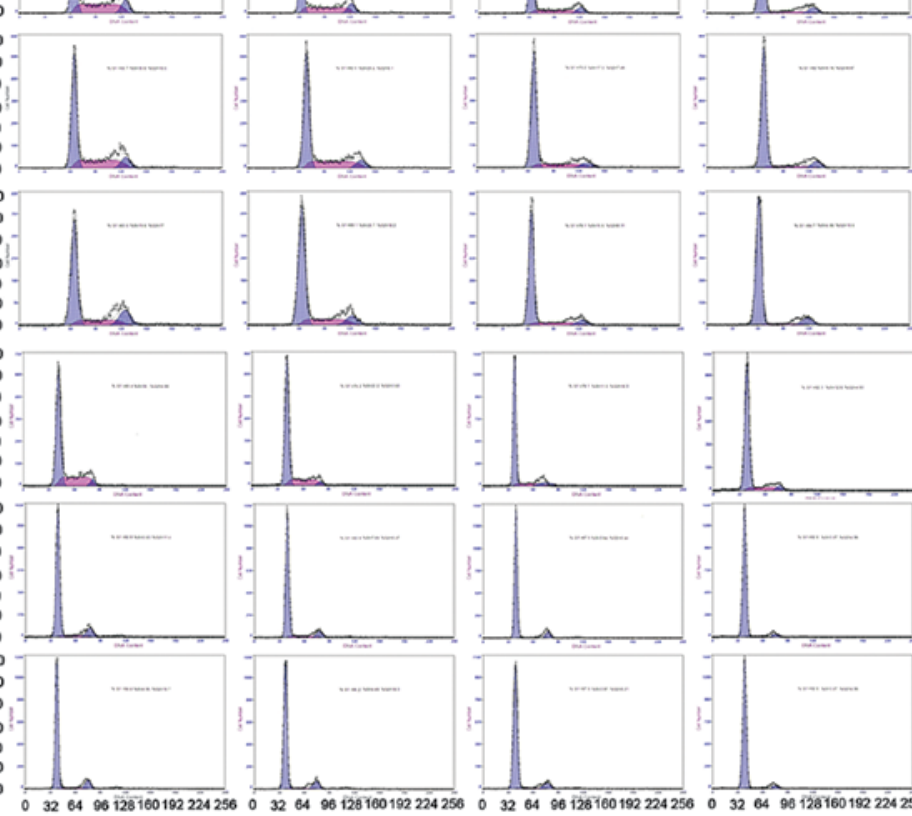

\section{DNA content}

B

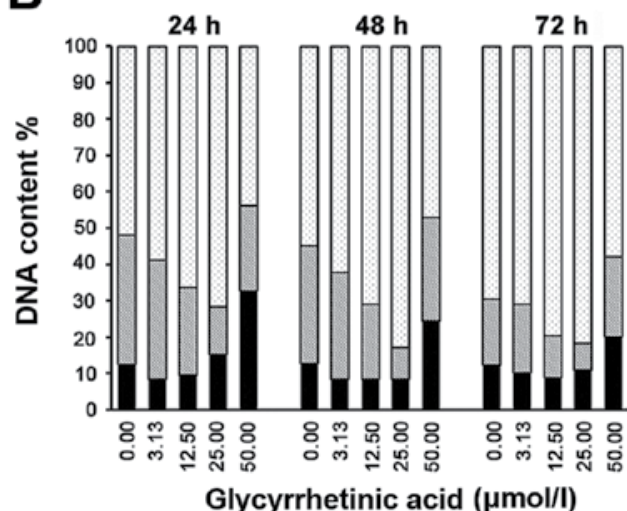

D

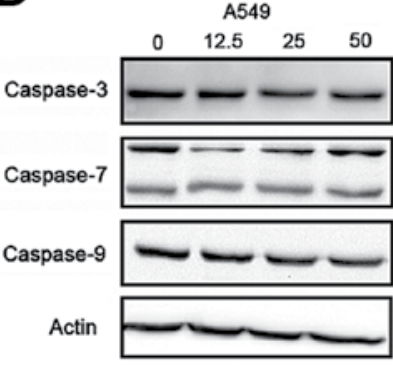

C
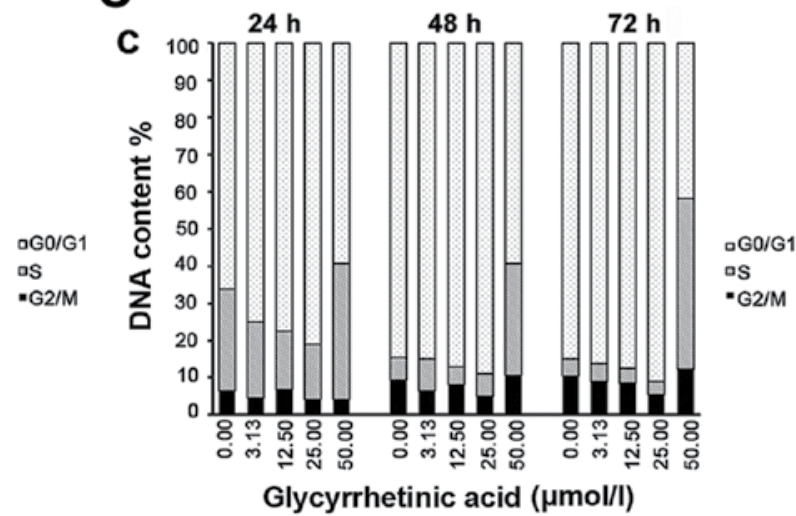

Glycyrrhetinic acid ( $\mu \mathrm{mol} / \mathrm{l})$

E

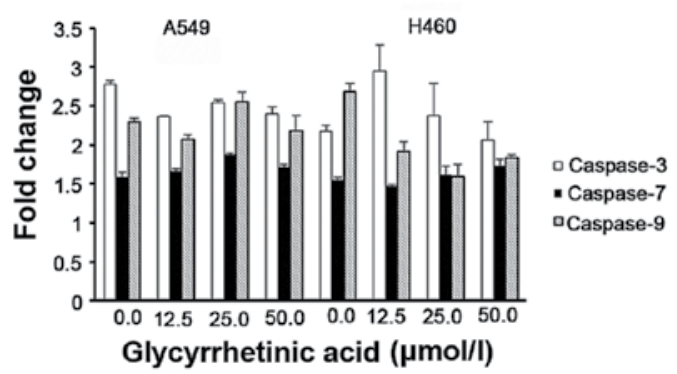

Figure 2. Glycyrrhetinic acid (GA) induces G1-phase cell cycle arrest in non-small cell lung cancer (NSCLC) cells without induction of apoptosis. Effect of GA on cell cycle was investigated using propidium iodide (PI) staining. Cells were treated with $0-50 \mu \mathrm{mol} / 1$ of GA for 24,48 and $72 \mathrm{~h}$, and then stained with PI Green peak represents G0/G1 phase, red peak represents S phase and blue peak represents G2 phase, respectively. Upper panel shows representative of three independent experiments with similar results, and lower panel represents the bar graphs of cells in different phases. Bar graph represents mean \pm SD from three independent experiments. (A) Cells were strikingly accumulated in the G1 phase after treatment with GA for 24, 48 and $72 \mathrm{~h}$. (B) Representative bar graph for A549 and (C) for NCI-H460 cells. (D) Western blot analysis of caspase-3, -7 and -9 protein expression after treatment with GA in a time (24 h)- and dose $(0,12.5,25,50 \mu \mathrm{mol} / 1)$-dependent manner. $\beta$-actin was used as a loading control. (E) Caspase-3, -7 and -9 activity in A549 and NCI-H460 cells treated with GA in a time (24h)- and dose $(0,12.5,25,50 \mu \mathrm{mol} / 1)$-dependent manner. Results shown are the mean of three independent experiments; error bars represent SD. GA-mediated G1-phase arrest is dependent on regulatory cyclin-dependent kinase inhibitors (CKIs) p18, p16, p27, p21, and GA decreases the levels of G1-phase regulatory CDKs and cyclins in both cell lines. 
with primary antibodies against caspase-3, -7 and -9, p18, p16, p27, p21, cyclin-D1, -D3 and -E2, CDK6, 4 and 2, E2F-1, $\mathrm{pRb}, \mathrm{Bip}, \mathrm{PERK}, \mathrm{ERP} 72$, or $\beta$-actin at $4^{\circ} \mathrm{C}$, and then incubated with HRP-conjugated secondary antibodies (anti-rabbit or mouse immunoglobulin $\mathrm{G}$ ) for $1 \mathrm{~h}$ at room temperature. Immunoreactivity was detected by ECL (Bio-Rad). $\beta$-actin was used as a loading control. Immunoblot experiments were repeated three times. Quantitative analysis was performed using Image Lab ${ }^{\mathrm{TM}}$ Software (Bio-Rad).

Statistical analysis. All values were expressed as mean \pm SD $(n=3)$. One-way analysis of variance (ANOVA) was used to determine statistical significance, followed by post hoc multiple comparisons (Dunn's test) using SPSS 19.0. $\mathrm{P}<0.05$ was considered to be statistically significant.

\section{Results}

GA suppresses the proliferation of NSCLC cells in vitro. To determine the suppression effect of GA (structure shown in Fig. 1A) on NSCLC cells, we performed a cell viability assay using A549 and NCI-H460 cell lines, respectively. After treatment for 24,48 or $72 \mathrm{~h}$, the viability of the two cell lines significantly decreased in a dose- and time-dependent manner (Fig. 1B and C).

GA arrests the cell cycle in G0/G1 phase. The results of flow cytometric analysis showed that the percentage of G0/G1 phase of both of A549 and H460 increased after treated with different concentrations of GA for 24, 48 and $72 \mathrm{~h}$ (Fig. 2A), respectively. Cell cycle distribution analysis showed that GA prevents the cell cycle progression by arresting the cells in the G0/G1 phase in both cell lines. In A549 cells, percentage of cells in G0/G1 phase increased from $52.03 \pm 1.42 \%$ (control group) to $71.63 \pm 6.61 \%$ for cells treated with $25 \mu \mathrm{mol} / \mathrm{l}$ of GA for $24 \mathrm{~h}$. At $48 \mathrm{~h}$, percentage of cells in $\mathrm{G} 0 / \mathrm{G} 1$ phase increased from $54.90 \pm 5.90 \%$ (control group) to $83.00 \pm 1.41 \%$ for cells treated with $25 \mu \mathrm{mol} / 1$ of GA. While at $72 \mathrm{~h}$, percentage of cells in G0/G1 phase increased from $69.70 \pm 5.38 \%$ (control group) to $81.83 \pm 2.58 \%$ for cells treated with $25 \mu \mathrm{mol} / 1$ of GA (Fig. 2A, upper panel). In NCI-H460, percentage of cells in the $\mathrm{G} 0 / \mathrm{G} 1$ phase increased from $66.10 \pm 0.99 \%$ (control group) to $80.95 \pm 1.91 \%$ for cells treated with $25 \mu \mathrm{mol} / \mathrm{l}$ of GA. At $48 \mathrm{~h}$, percentage of cells in G0/G1 phase increased from $84.60 \pm 2.40 \%$ (control group) to $88.95 \pm 2.19 \%$ for cells treated with $25 \mu \mathrm{mol} / 1$ of GA. While at $72 \mathrm{~h}$, percentage of cells in the G0/G1 phase increased from $85.00 \pm 0.85 \%$ (control group) to $91.00 \pm 2.26 \%$ for cells treated with $25 \mu \mathrm{mol} / 1$ of GA (Fig. 2B, upper panel). No increase in $\mathrm{S}$ or $\mathrm{G} 2 / \mathrm{M}$ peak was observed in either cell line.

Taken together, our data strongly suggested that GA did not induce apoptosis but caused cell cycle arrest in G0/G1 phase in NSCLC cells. Annexin assay did not show any significant changes in apoptotic/necrotic cell population for all concentrations of GA as compared to the control group in either cell line at 24,48 and $72 \mathrm{~h}$, respectively. To further validate the above data, we checked the expression levels of caspase-3, -7 and -9 in both cell lines after $24 \mathrm{~h}$ treatment with GA by western blot analysis. Expression of caspase-3 decreased with increase in drug concentration in NCI-H460 cells, but no significant changes in caspase-7 and -9 protein levels or activity were observed in A549 or NCI-H460 cells (Fig. 2D and E), suggesting that GA did not hinder the viability of cells.

GA downregulates the levels of cell cycle regulatory proteins and retinoblastoma $(R b)$ phosphorylation. To investigate the causes of cell cycle arrest, cyclin-dependent kinase inhibitors (CKIs), such as p27, p21, p18 and p16 that regulate $\mathrm{G} 0 / \mathrm{G} 1$ phase of cell cycle progression were examined by western blot analysis (19-21). In A549 cells, the levels of p27, p21, p18 and p16 were significantly increased after $24 \mathrm{~h}$ treatment with GA as compared to the control cells (Fig. 3C). H460 cells also showed similar results (Fig. 3D). To further dissect the biochemical events controlling the transition of cell cycle phases, we examined the levels of several proteins, such as cyclin-D1, -D3 and -E2, CDK4, 6 and 2, which are involved in G0/G1-phase progression, and found that GA significantly decreased the expression of these proteins in both cell lines (Fig. 3E and F). GA also significantly decreased the expression levels of E2F-1, the essential transcription factor that regulates cell cycle progression and apoptosis, and pRb (Fig. 3G and H).

GA upregulated the levels of ER stress regulatory proteins. Previous studies have demonstrated that ER stress triggers G1-phase cell cycle arrest in various cancer cells (22). Therefore, we examined whether ER stress was induced by GA. Bip is the master regulator of ER function. Phosphorylation of double-stranded RNA-activated PERK is closely associated with Bip. Hence, we checked the expression of Bip, PERK and ERP72 in both cell lines by western blot analysis, and found that the expression levels of these proteins were significantly upregulated after $24 \mathrm{~h}$ treatment with GA (Fig. 4A and B).

\section{Discussion}

GA is a natural active compound that is extracted from the Chinese herbal medicine glycyrrhiza. GA was shown to induce cell cycle arrest in G1 phase $(13,15)$. The role of GA in NSCLC, especially its relationship with ER stress has not been reported. In the present study, we found that GA induced G0/G1 arrest in A549 and NCI-H460 cell lines, which provides a useful model system to characterize the cytotoxic effects of therapeutic agents. Furthermore, GA could have therapeutic potential in the treatment of NSCLC.

Our results have shown that GA successfully inhibited proliferation of two NSCLC cell lines, A549 and NCI-H460. Cell cycle analysis by flow cytometry showed that GA induced a modest increase in G0/G1 phase in both cell lines. However, based on expression levels of caspase-3, -7 and -9 by western blot analysis, GA did not induce apoptosis in either cell line.

It is well known that eukaryotic cell cycle is regulated by the coordinated activity of protein kinase complexes, each consisting of a cyclin-dependent kinase (CDK) and cyclins. CDK complexes are formed and activated at specific stages of the cell cycle, and their activities are required for progression through distinct cell cycle phases (23). Progression through G1 phase requires the activities of cyclin-D-dependent CDK4 or 6 , followed by activation of the cyclin-E- and cyclin-A-dependent kinase CDK2. The cyclin-CDK complex 
A

A549
Glycyrrhetinic acid $(\mu \mathrm{mol} / \mathrm{l})$

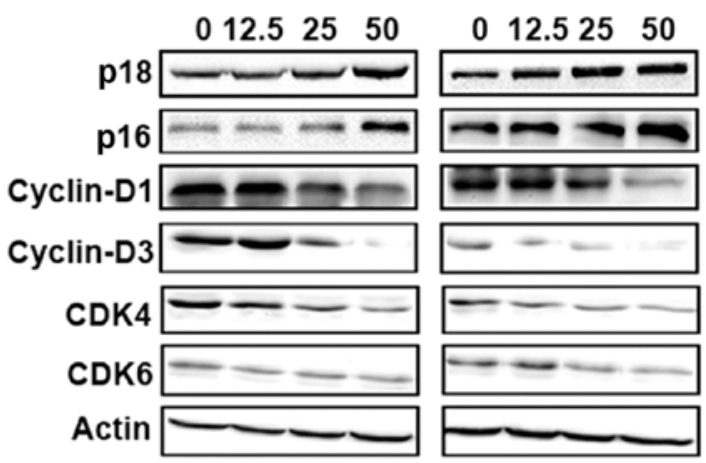

C

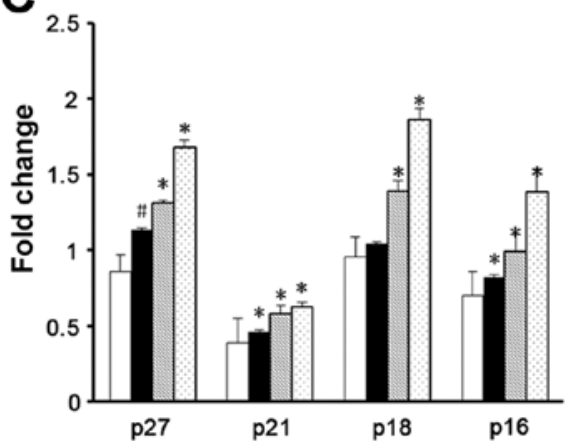

$\mathbf{E}$

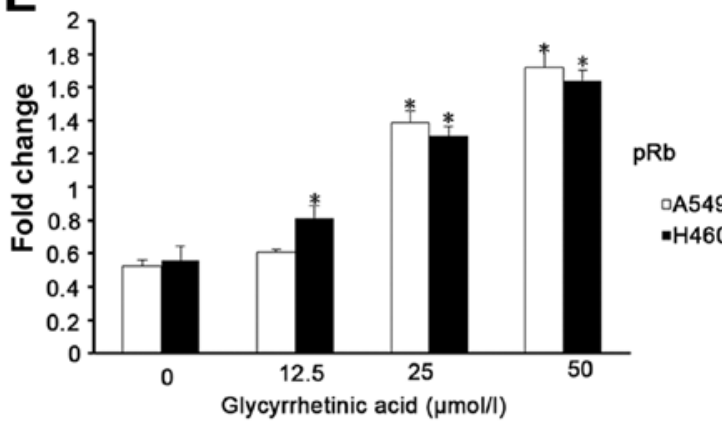

B

A549

Glycyrrhetinic acid ( $\mu \mathrm{mol} / \mathrm{l})$

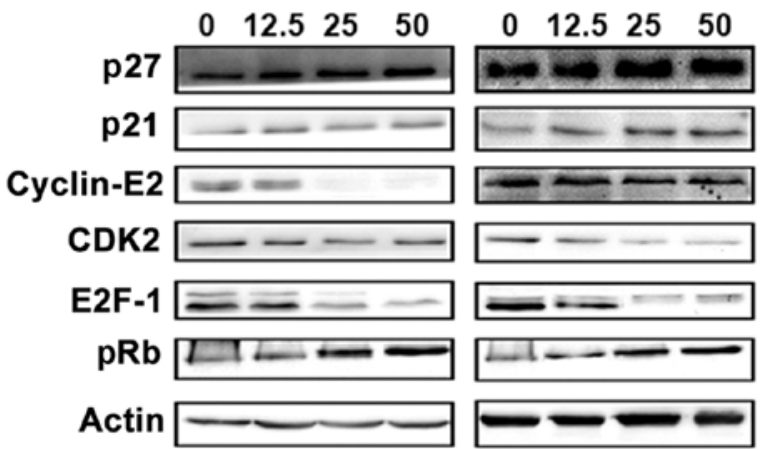

D

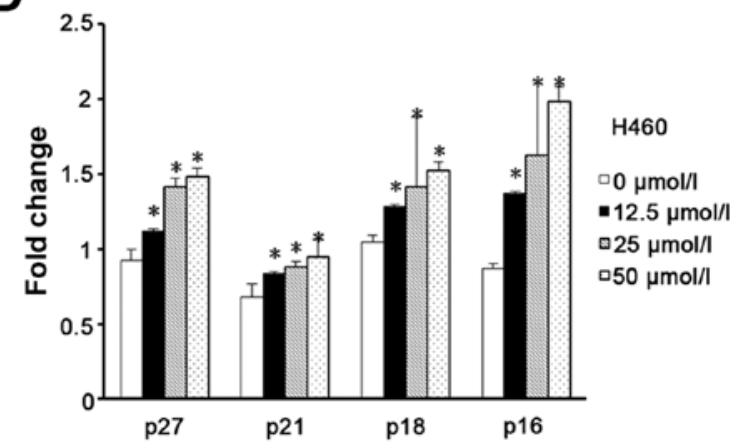

$\mathbf{F}$

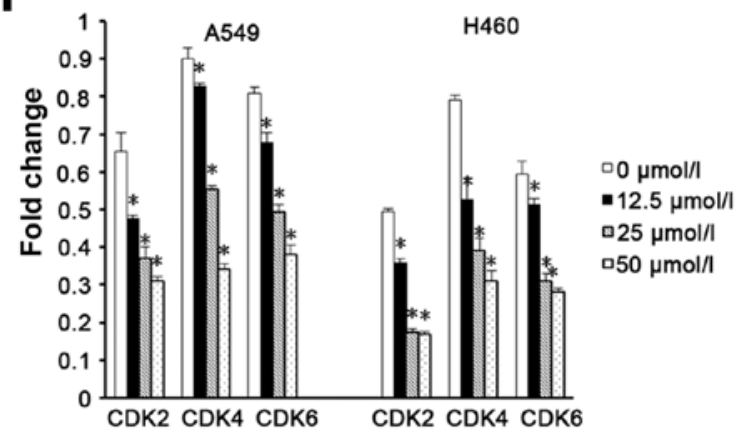

G

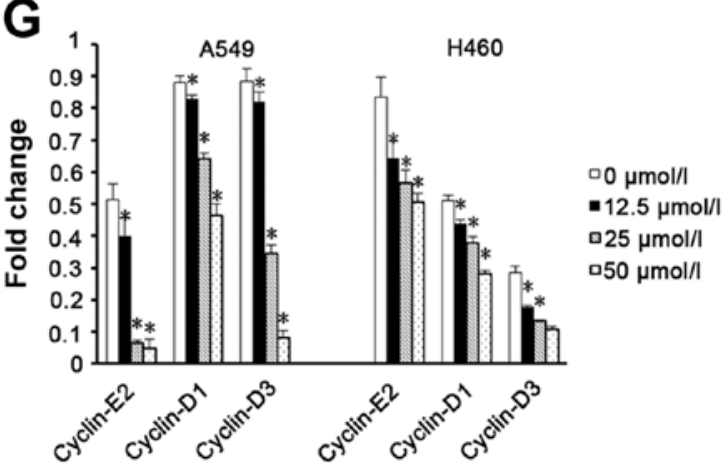

H

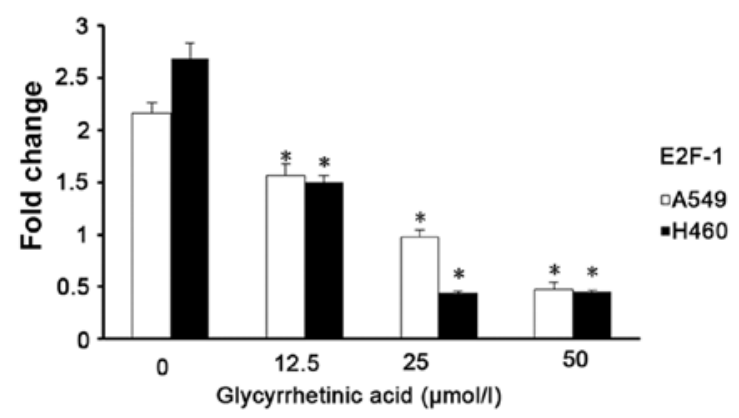

Figure 3. Glycyrrhetinic acid (GA) affects cell cycle regulatory proteins, E2F-1 and pRb. (A and B) Effect of GA on G1-phase regulatory cyclin-dependent kinase inhibitors (CKIs), cyclins, CDKs, E2F-1 and pRb assessed by western blot analysis in A549 and NCI-H460 cells treated with various concentrations of GA $(12.5,25$ and $50 \mu \mathrm{mol} / \mathrm{l})$ for $24 \mathrm{~h}$. $\beta$-actin was used as a loading control. (C-H) Densitometry analysis of CKIs, cyclins, CDKs, E2F-1 and pRb levels relative to $\beta$-actin were performed using three independent experiments. Error bars represent $\mathrm{SD} ;{ }^{*} \mathrm{p}<0.01,{ }^{\sharp} \mathrm{p}<0.05$. GA induces endoplasmic reticulum (ER) stress by activating Bip in both cell lines.

formed during G1-phase catalyses phosphorylation of the dominant inhibitor of G1/S-cell cycle progression, the Rb family of tumor suppressor proteins, thereby blocking their inhibitory activity allowing the cell to progress into S phase (24-27). It is also known that these cyclin-CDK complexes often bind to CKIs including p16, p18, p21 and p27, which inhibit their 


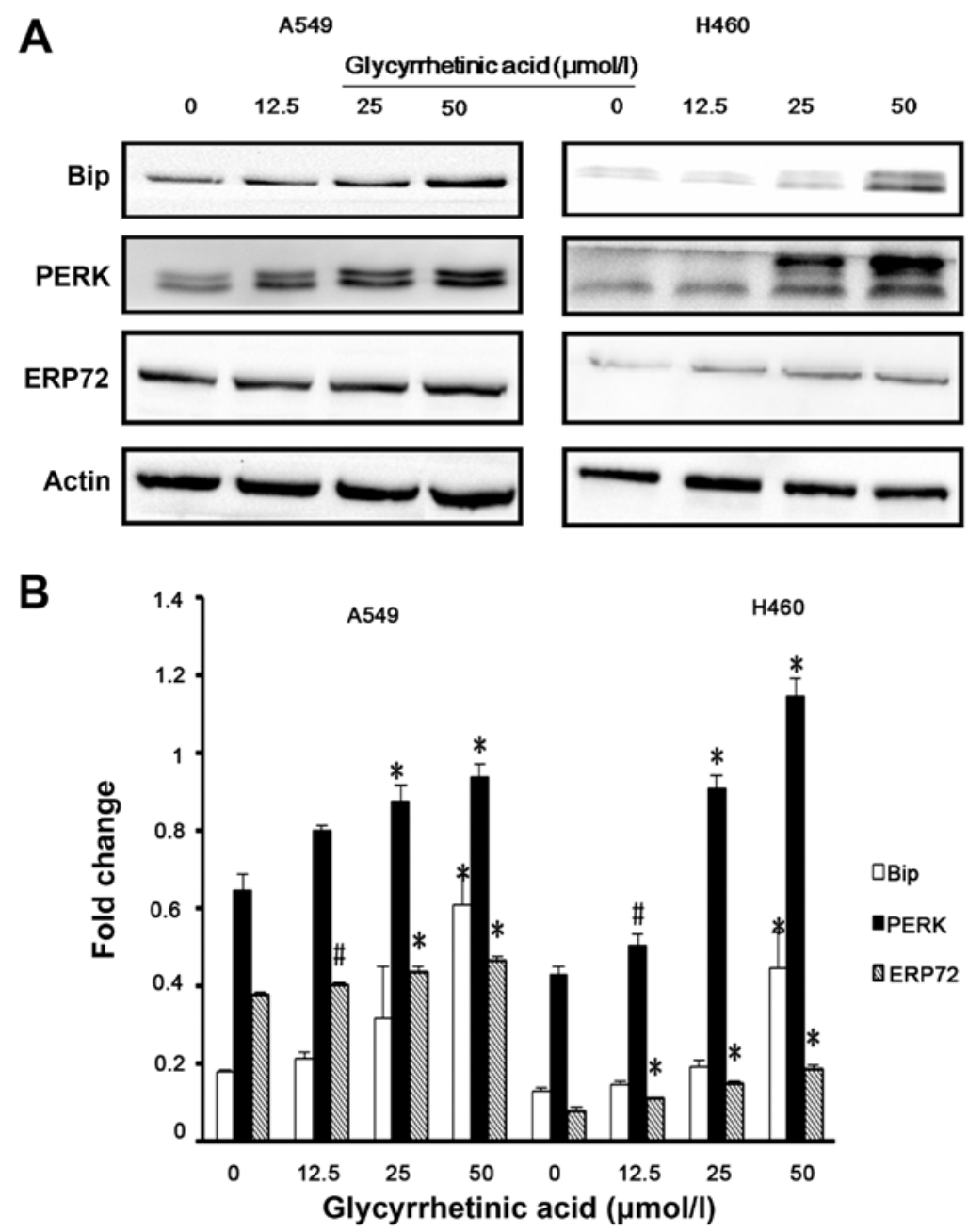

Figure 4. Glycyrrhetinic acid (GA) induces endoplasmic reticulum (ER) stress. (A) A549 and NCI-H460 cells treated with various concentrations of GA (12.5, 25 and $50 \mu \mathrm{mol} / 1)$ for $24 \mathrm{~h}$ were analyzed by immunoblotting with antibodies against Bip, protein kinase-like ER kinase (PERK) and ERP72. $\beta$-actin was used as a loading control. (B) Densitometry analysis of Bip, PERK and ERP72 levels relative to $\beta$-actin were performed using three independent experiments. Error bars represent SD; ${ }^{\mathrm{p}} \mathrm{B}<0.01,{ }^{\sharp} \mathrm{p}<0.05$.

kinase activities, and prevent cell cycle progression (28). E2F-1 is an essential transcription factor that regulates cell cycle progression and cell proliferation. E2F-1 activity is regulated by the $\mathrm{Rb}$ protein that binds activator E2F proteins to inhibit transcription outside of G1/S in animals (29).

Flow cytometric analysis of A549 and NCI-H460 cells treated with GA showed that GA inhibits cell cycle progression by blocking the transition from $\mathrm{G} 1$ to $\mathrm{S}$ phase. To further investigate this result, western blot analysis was used to examine proteins associated with the cell cycle, e.g., cyclin-D1/D3, which is expressed in G1 phase and binds to CDK4 and 6 to activate them, followed by activation of the cyclin-E-dependent kinase CDK2. These protein kinase complexes were inhibited by GA. GA also significantly decreased the expression levels of E2F-1 and pRb in both cell lines. Our results indicated that GA induced growth inhibition mainly via regulation of $\mathrm{p} 16$, p18, p21 and p27 status in A549 and NCI-H460 cells.

In the present study, the analysis of DNA content versus light scatter of the GA-treated A549 and NCI-H460 cells indicated no apoptosis. Similarly, the expression of caspase- $-3,-7$ and -9 measured by western blot analysis indicated that GA could not induce apoptosis in these cells. However, GA induced expression of ER proteins GRP78/Bip, PERK and ERP72, which are associated with ER stress. This result suggested that GA inhibited proliferation of A549 and NCI-H460 cells and caused G0/G1-phase cell cycle arrest via ER stress rather than apoptosis.

GRP78/Bip is a major cellular target of the UPR, an ER chaperone that not only binds to unfolded proteins but also regulates the activation of ER stress transducers such as IRE1, PERK, and ATF6 (30-32). GRP78/Bip is ubiquitously expressed at very low levels in growing cells, but it is highly expressed in response to numerous cellular stresses. ERP72, a member of the protein disulfide isomerases (PDI) family, is localized in the ER, and plays a major role in quality control and folding (33). Dysregulation of ER chaperone/folding enzymes ERP72 and GRP78/Bip occurred early after ablation of PERK function suggesting that changes in ER secretory functions could reduce insulin gene expression and cell proliferation $(34,35)$.

Previous studies have found that CKIs and cyclins play important roles in ER stress and cell cycle arrest. p27 was reported to be a critical mediator of ER stress-induced G1 cell cycle arrest in melanoma cells (36). p21 integrates the DNA damage response with ER stress signaling, which 


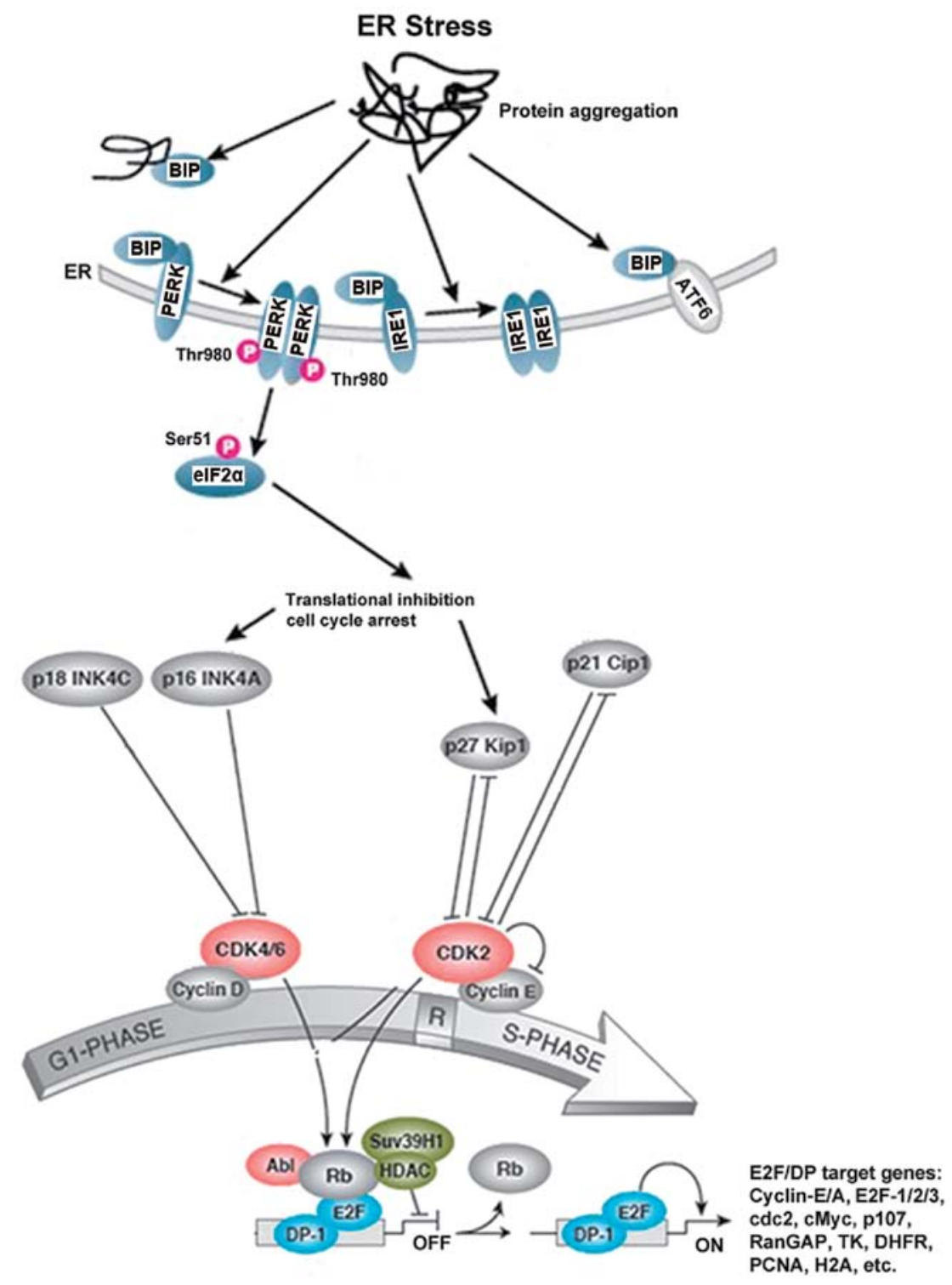

Figure 5. The proposed mechanism of action of glycyrrhetinic acid (GA)-induced G1-phase arrest in non-small cell lung cancer (NSCLC) cells. The G1/S cell cycle checkpoint controls the passage of eukaryotic cells from the first phase (G1) into the DNA synthesis phase (S). Two cell cycle kinases, CDK4/6-cyclin-D and CDK2-cyclin-E, and the transcription complex that includes retinoblastoma $(\mathrm{Rb})$ and E2F are pivotal in controlling this checkpoint. During G1 phase, the Rb-HDAC repressor complex binds to the E2F-DP-1 transcription factors, inhibiting downstream transcription. Phosphorylation of Rb by CDK4/6 and CDK2 dissociates the Rb-repressor complex, permitting transcription of S-phase genes encoding for proteins that amplify the G1- to S-phase switch, and are required for DNA replication. Many different stimuli exert checkpoint control including endoplasmic reticulum (ER) stress, TGF $\beta$, DNA damage, contact inhibition, replicative senescence and growth factor withdrawal. ER stress is induced by members of the INK4 (p16, p18) and Kip/Cip (p21, p27) families of cell cycle kinase inhibitors. The accumulation of unfolded proteins in the ER triggers the unfolded protein response (UPR), which is a stress signaling pathway. The UPR coordinates the induction of ER chaperones, decreases protein synthesis, and causes growth arrest in G1 phase of the cell cycle.

then regulates mitochondrial death pathways during chronic genotoxic stress (37). Translational regulation of cyclin-D1 in response to ER stress is a mechanism for checkpoint control that prevents cell cycle progression (17). PERK has been shown to mediate cell cycle arrest by blocking cyclin-D1 translation during UPR $(17,38)$. Similarly, our study has shown that induction of members of the INK4 (p16, p18) or Kip/Cip (p21, p27) families of cell cycle kinase inhibitors causes ER stress, and accumulation of unfolded proteins in the ER triggers UPR, which is a stress signaling pathway. The UPR coordinates the induction of ER chaperones with decreased protein synthesis and growth arrest in G1 phase of the cell cycle. Based on our results, we propose a model for the mechanism of action of GA in NSCLC cells (Fig. 5).
We have convincingly shown that GA inhibits proliferation of NSCLC cell lines by causing cell cycle arrest in G0/G1 phase in a time- and dose-dependent manner, without inducing apoptosis. We have elucidated a new mechanism of action of GA against NSCLC by inducing G1-phase cell cycle arrest through ER stress pathway. Since GA synergizes the effect of anticancer drugs, it provides new insight into the therapeutic index of NSCLC treatment.

\section{Acknowledgements}

This study was supported by the National Science and Technology Pillar Program in the 11th Five-year Plan of China 2006BAI11B08-01 (to H.F. and X.Z.), the U.S. National 
Institutes of Health grants P01 CA116676 (to H.F.), the Priority Academic Program Development (PAPD) of Jiangsu Higher Education Institutions (to X.Z.), China and Europe taking care of healthcare solutions, CHETCH Grant Agreement Number: PIRSES-GA-2013-612589 (to X.Z.) and the Natural Science Foundation of Jiangsu Province (BK20131415) (to M.C.).

\section{References}

1. Parkin DM, Bray F, Ferlay J and Pisani P: Global cancer statistics, 2002. CA Cancer J Clin 55: 74-108, 2005.

2. Jemal A, Siegel R, Ward E, Murray T, Xu J and Thun MJ: Cancer statistics, 2007. CA Cancer J Clin 57: 43-66, 2007.

3. Wang T, Nelson RA, Bogardus A and Grannis FW Jr: Five-year lung cancer survival: which advanced stage nonsmall cell lung cancer patients attain long-term survival? Cancer 116: 1518-1525, 2010.

4. Carrera S, Buque A, Azkona E, et al: Epidermal growth factor receptor tyrosine-kinase inhibitor treatment resistance in non-small cell lung cancer: biological basis and therapeutic strategies. Clin Transl Oncol 16: 339-350, 2014.

5. Agarwal MK, Iqbal M and Athar M: Inhibitory effect of 18beta-glycyrrhetinic acid on 12-O-tetradecanoyl phorbol13-acetate-induced cutaneous oxidative stress and tumor promotion in mice. Redox Rep 10: 151-157, 2005.

6. Jeong HG, You HJ, Park SJ, et al: Hepatoprotective effects of 18beta-glycyrrhetinic acid on carbon tetrachloride-induced liver injury: inhibition of cytochrome P450 2E1 expression. Pharmacol Res 46: 221-227, 2002.

7. Matsui S, Matsumoto H, Sonoda Y, et al: Glycyrrhizin and related compounds down-regulate production of inflammatory chemokines IL-8 and eotaxin 1 in a human lung fibroblast cell line. Int Immunopharmacol 4: 1633-1644, 2004.

8. Abe N, Ebina T and Ishida N: Interferon induction by glycyrrhizin and glycyrrhetinic acid in mice. Microbiol Immunol 26 : 535-539, 1982.

9. Dai JH, Iwatani Y, Ishida T, et al: Glycyrrhizin enhances interleukin-12 production in peritoneal macrophages. Immunology 103 235-243, 2001.

10. Ukil A, Biswas A, Das T and Das PK: 18 Beta-glycyrrhetinic acid triggers curative Th1 response and nitric oxide up-regulation in experimental visceral leishmaniasis associated with the activation of NF-kappa B. J Immunol 175: 1161-1169, 2005.

11. Lee CS, Yang JC, Kim YJ, Jang ER, Kim W and Myung SC: $18 \beta$-glycyrrhetinic acid potentiates apoptotic effect of trichostatin A on human epithelial ovarian carcinoma cell lines. Eur J Pharmacol 649: 354-361, 2010.

12. Kuang $\mathrm{P}$, Zhao W, Su W, et al: $18 \beta$-glycyrrhetinic acid inhibits hepatocellular carcinoma development by reversing hepatic stellate cell-mediated immunosuppression in mice. Int $J$ Cancer 132: 1831-1841, 2013.

13. Satomi Y, Nishino H and Shibata S: Glycyrrhetinic acid and related compounds induce G1 arrest and apoptosis in human hepatocellular carcinoma HepG2. Anticancer Res 25: 4043-4047, 2005.

14. Sharma G, Kar S, Palit S and Das PK: $18 \beta$-glycyrrhetinic acid induces apoptosis through modulation of Akt/FOXO3a/Bim pathway in human breast cancer MCF-7 cells. J Cell Physiol 227: 1923-1931, 2012

15. Wang D, Wong HK, Feng YB and Zhang ZJ: 18Beta-glycyrrhetinic acid induces apoptosis in pituitary adenoma cells via ROS/MAPKs-mediated pathway. J Neurooncol 116: 221-230, 2014.

16. Lin KW, Huang AM, Hour TC, Yang SC, Pu YS and Lin CN: $18 \beta$-glycyrrhetinic acid derivatives induced mitochondrial-mediated apoptosis through reactive oxygen species-mediated p53 activation in NTUB1 cells. Bioorg Med Chem 19: 4274-4285, 2011.

17. Brewer JW, Hendershot LM, Sherr CJ and Diehl JA: Mammalian unfolded protein response inhibits cyclin D1 translation and cell-cycle progression. Proc Natl Acad Sci USA 96: 8505-8510, 1999.
18. Puthalakath H, O'Reilly LA, Gunn P, et al: ER stress triggers apoptosis by activating BH3-only protein Bim. Cell 129: 1337-1349, 2007.

19. Chu IM, Hengst L and Slingerland JM: The Cdk inhibitor p27 in human cancer: prognostic potential and relevance to anticancer therapy. Nat Rev Cancer 8: 253-267, 2008.

20. Pérez-Sayáns M, Suárez-Peñaranda JM, Gayoso-Diz P, Barros-Angueira F, Gándara-Rey JM and García-García A: The role of $\mathrm{p} 21 \mathrm{Waf} 1 / \mathrm{CIP} 1$ as a Cip/Kip type cell-cycle regulator in oral squamous cell carcinoma (Review). Med Oral Patol Oral Cir Bucal 18: e219-e225, 2013.

21. Drexler HG: Review of alterations of the cyclin-dependent kinase inhibitor INK4 family genes p15, p16, p18 and p19 in human leukemia-lymphoma cells. Leukemia 12: 845-859, 1998.

22. Zhu GY, Wong BC, Lu A, et al: Alkylphenols from the roots of Ardisia brevicaulis induce G1 arrest and apoptosis through endoplasmic reticulum stress pathway in human non-small-cell lung cancer cells. Chem Pharm Bull (Tokyo) 60: 1029-1036, 2012.

23. Sherr CJ: Cancer cell cycles. Science 274: 1672-1677, 1996.

24. Sherr CJ: D-type cyclins. Trends Biochem Sci 20: 187-190, 1995

25. Guadagno TM and Newport JW: Cdk2 kinase is required for entry into mitosis as a positive regulator of Cdc2-cyclin B kinase activity. Cell 84: 73-82, 1996

26. Geng Y, Whoriskey W, Park MY, et al: Rescue of cyclin D1 deficiency by knockin cyclin E. Cell 97: 767-777, 1999.

27. Jin YH, Choi J, Shin S, Lee KY, Park JH and Lee SK: Panaxadiol selectively inhibits cyclin A-associated Cdk2 activity by elevating p21WAF1/CIP1 protein levels in mammalian cells. Carcinogenesis 24: 1767-1772, 2003

28. Grana X and Reddy EP: Cell cycle control in mammalian cells: role of cyclins, cyclin dependent kinases (CDKs), growth suppressor genes and cyclin-dependent kinase inhibitors (CKIs). Oncogene 11: 211-219, 1995.

29. Bertoli C, Skotheim JM and de Bruin RA: Control of cell cycle transcription during G1 and S phases. Nat Rev Mol Cell Biol 14 518-528, 2013.

30. Harding HP, Zhang Y, Zeng H, et al: An integrated stress response regulates amino acid metabolism and resistance to oxidative stress. Mol Cell 11: 619-633, 2003.

31. Shi K, Wang D, Cao X and Ge Y: Endoplasmic reticulum stress signaling is involved in mitomycin C (MMC)-induced apoptosis in human fibroblasts via PERK pathway. PLoS One 8: e59330, 2013.

32. Yoon JS, Kim HM, Yadunandam AK, et al: Neferine isolated from Nelumbo nucifera enhances anti-cancer activities in Hep3B cells: molecular mechanisms of cell cycle arrest, ER stress induced apoptosis and anti-angiogenic response. Phytomedicine 20: 1013-1022, 2013.

33. Mazzarella RA, Srinivasan M, Haugejorden SM and Green M: ERp72, an abundant luminal endoplasmic reticulum protein, contains three copies of the active site sequences of protein disulfide isomerase. J Biol Chem 265: 1094-1101, 1990.

34. Brewer JW and Diehl JA: PERK mediates cell-cycle exit during the mammalian unfolded protein response. Proc Natl Acad Sci USA 97: 12625-12630, 2000.

35. Feng D, Wei J, Gupta S, McGrath BC and Cavener DR: Acute ablation of PERK results in ER dysfunctions followed by reduced insulin secretion and cell proliferation. BMC Cell Biol 10: 61, 2009.

36. Han C, Jin L, Mei Y and Wu M: Endoplasmic reticulum stress inhibits cell cycle progression via induction of p27 in melanoma cells. Cell Signal 25: 144-149, 2013.

37. Vitiello PF, Wu YC, Staversky RJ and O'Reilly MA: p21(Cip1) protects against oxidative stress by suppressing ER-dependent activation of mitochondrial death pathways. Free Radic Biol Med 46: 33-41, 2009.

38. Hamanaka RB, Bennett BS, Cullinan SB and Diehl JA: PERK and GCN2 contribute to eIF2alpha phosphorylation and cell cycle arrest after activation of the unfolded protein response pathway. Mol Biol Cell 16: 5493-5501, 2005. 